\title{
The Inter-relationships between Vegetarianism and Eating Disorders among Females
}

\author{
Anna M. Bardone-Cone, PhD [associate professor], \\ Department of Psychology, University of North Carolina at Chapel Hill, Chapel Hill, NC; \\ Department of Psychological Sciences, University of Missouri, Columbia, MO. \\ Ellen E. Fitzsimmons-Craft, MA [doctoral student], \\ Department of Psychology, University of North Carolina at Chapel Hill, Chapel Hill, NC
}

Megan B. Harney, MA [doctoral student], Department of Psychology, University of North Carolina at Chapel Hill, Chapel Hill, NC

Christine R. Maldonado, PhD [adjunct professor], Department of Psychology, Boise State University, Boise, ID.

Melissa A. Lawson, MD [associate clinical professor], University of Missouri School of Medicine, Columbia, MO.

Roma Smith, LPN [senior LPN], and University of Missouri School of Medicine, Columbia, MO.

D. Paul Robinson, MD [associate clinical professor]

Florida State University School of Medicine, Tallahassee, FL.

\section{Abstract}

When individuals with a suspected or diagnosed eating disorder adopt a vegetarian diet, health care professionals may worry that this choice may function as a socially acceptable way to legitimize food avoidance. Yet, limited research has examined vegetarianism in relation to eating disorders. Study objectives were to compare individuals with and without an eating disorder history, and individuals at different stages of eating disorder recovery, on past and current vegetarianism and motivations for and age at becoming vegetarian. Participants were females seen at some point for an eating disorder $(n=93)$ as well as controls who never had an eating disorder ( $n=67)$. Recruitment and data collection for this cross-sectional study occurred in 2007-2008. Chi square analyses and analyses of variance and covariance were used to examine the research questions. Compared to controls, individuals with an eating disorder history were significantly more likely to ever have been vegetarian ( $52 \%$ vs. $12 \%)$, to be currently vegetarian ( $24 \%$ vs. $6 \%$ ), and to be primarily motivated by weight-related reasons ( $42 \%$ vs. $0 \%)$. The three recovery status groups (fully recovered, partially recovered, active eating disorder) did not differ significantly in percentiles endorsing a history of vegetarianism or weight-related reasons as primary, but they differed significantly in current vegetarianism (33\% of active cases, $13 \%$ of partially recovered, $5 \%$ of fully recovered). Most perceived that their vegetarianism was related to their eating disorder

(C) 2012 Academy of Nutrition and Dietetics. Published by Elsevier Inc. All rights reserved.

Address correspondence to: Anna M. Bardone-Cone, PhD, CB \#3270 Davie Hall, Department of Psychology, University of North Carolina at Chapel Hill, Chapel Hill, NC 27599. bardonecone@unc.edu.

Publisher's Disclaimer: This is a PDF file of an unedited manuscript that has been accepted for publication. As a service to our customers we are providing this early version of the manuscript. The manuscript will undergo copyediting, typesetting, and review of the resulting proof before it is published in its final citable form. Please note that during the production process errors may be discovered which could affect the content, and all legal disclaimers that apply to the journal pertain. 
(68\%) and emerged after its onset. Results shed light on the vegetarianism-eating disorders relation and suggest intervention considerations for clinicians (e.g., investigating motives for vegetarianism).

\section{Keywords}

vegetarianism; eating disorders; recovery

\section{INTRODUCTION}

Following a vegetarian diet is often associated with health-related benefits such as a decreased likelihood of cardiovascular disease $(1,2)$. However, when a patient with a suspected or diagnosed eating disorder becomes vegetarian, health care professionals may view the behavior with skepticism and as a red flag. Researchers have suggested that vegetarianism may be used by some as a socially acceptable way to legitimize food avoidance and avoid certain eating situations (3) and as a "smokescreen for more severe eating pathology" (4). A vegetarian lifestyle may also further simplify the lives of individuals with eating pathology in terms of providing clear do's and don'ts of eating, which may facilitate efforts at restriction. However, little empirical data exist regarding vegetarianism and eating disorders and only two studies have considered vegetarianism in relation to recovery from an eating disorder $(5,6)$.

Evidence suggests that among patients with anorexia nervosa (AN), about 50\% report eating some form of vegetarian diet (45-54\%) (7,8), compared to about 6-34\% of adolescent and young adult women overall $(3,9,10)$. One study found that about half of their participants with AN reported having become vegetarian several years prior to the onset of the eating disorder (11), while another study found this to be true for only a small minority of the vegetarians with AN (6\%) (8). Thus, the temporal ordering between the onset of an eating disorder and the onset of vegetarianism is unclear. While motivations for vegetarianism of health, weight loss/maintenance, and animal ethics are consistently cited by young female vegetarians $(3,9,12)$, no studies were found comparing reasons for becoming vegetarian in eating disorder and non-eating disorder samples. Regarding the recovery process, researchers have found that more non-remitted AN patients than remitted AN patients were vegetarian, that vegetarianism significantly contributed to non-remission from $\mathrm{AN}$, and that vegetarianism was still prominent in individuals "recovered" from AN $(5,6)$. Of note, past work on eating disorder recovery and vegetarianism has not used comprehensive definitions of recovery which include a psychological aspect of recovery and yield greater recovery stability (13).

The primary objective of this study was to understand rates of vegetarianism (both past and current), motivations for vegetarianism, and age at becoming vegetarian in two sets of analyses related to eating disorders. First, women with a history of an eating disorder were compared to those with no history of an eating disorder on these aspects of vegetarianism. Second, comparisons occurred for women at different stages of recovery from an eating disorder: full recovery, partial recovery, and active eating disorder. As a secondary objective, for those with a history of an eating disorder, age at becoming vegetarian was compared to age of onset of eating disorder symptoms, and individuals' perceptions about the relationship between vegetarianism and their eating disorder were investigated. Given the exploratory nature of this study, hypotheses are limited to the following: individuals with a history of an eating disorder will have higher rates of vegetarianism and their vegetarianism will more likely have been motivated by weight-related reasons compared to those with no history of an eating disorder. 


\section{METHODS}

\section{Participants and Recruitment}

Attempts were made to contact all female eating disorder patients (ages 16 and older) seen at the University of Missouri Pediatric and Adolescent Specialty Clinic $(N=273)$ between 1996 and 2007. This clinic is a primary care and referral clinic specializing in the care of children and adolescents (ages 10-25 years) that has physicians with eating disorders expertise. Of the 273 eating disorder patients, $96(35.2 \%)$ were successfully contacted and recruited. Fifty-five $(20.1 \%)$ of the 273 were contacted but did not participate due to other time commitments or lack of interest. Of the remaining patients, four $(1.5 \%)$ were deceased and 118 patients (43.2\%) could not be contacted due to absent or incorrect mailing addresses or inability to make phone contact. Prior studies following up eating disorder patients over a range of about 10 years have reported similar rates of contact and participation $(5,14)$, and results indicated that participants in the current study were not significantly different from non-participants on eating disorder diagnoses or age at first clinic visit. They also did not differ at first clinic visit in body mass index (BMI), which results from dividing weight in kilograms by height in meters squared and can be considered a measure of relative weight. In sum, of the 151 eating disorder patients contacted, $63.6 \%$ participated.

Non-eating disorder controls were recruited from two sources: the clinic from which the eating disorder patients were recruited $(n=17)$ and a convenience sample from the university campus $(n=50)$. The sample from the clinic encompassed individuals identified by physicians as not having an eating disorder or other chronic condition. The sample from the university was recruited via campus fliers and psychology classes. Eligible controls were females ages 16 and older with no current or past eating disorder symptoms. Absence of an eating disorder history was determined by screener questions administered via phone and the eating disorder module of the Structured Clinical Interview for DSM-IV (SCID) (15) administered in person; women were eligible as controls if both the screener and interview indicated no history of an eating disorder, threshold or subthreshold.

\section{Study Procedures}

Participants first completed a set of questionnaires and then, typically one week later, an interview including a diagnostic clinical interview. Recruitment and data collection for this cross-sectional study occurred over 18 months in 2007-2008. Most participants completed the interview in person (about $70 \%$ of the eating disorder sample and $100 \%$ of the controls); those who lived too far away to travel to the study site did a phone interview (e.g., former eating disorder patients who had moved out of state). Participants were provided financial remuneration (or, for psychology students, course credit) after completing the interview. All aspects of this study were approved by the University of Missouri Institutional Review Board, including obtaining written consent and, in the case of minors, parental written consent and the child's assent.

\section{Measures}

Measures related to vegetarianism-In the questionnaire portion of the study, participants were asked: "Have you ever considered yourself a type of vegetarian (e.g., not eating beef but eating other meat; not eating any meat at all)?" with response options of "yes" and "no." Most of the research on vegetarianism refers to vegetarianism broadly defined, with "semi-vegetarians" (e.g., those who may eat fish and/or chicken but no red meat) grouped together with strict vegetarians (i.e., those who do not eat any meat) (16); this is the approach used in the current study. Those who replied affirmatively to having ever been vegetarian were asked the age at which they first became some type of vegetarian, as well as the primary reason for cutting out meat from their diet with options of: "health 
reasons - e.g., it is healthier not to eat meat (or not to eat certain kinds of meat)," "ethical reasons - e.g., it is wrong to eat animals; concerns about conditions animals are kept in," "weight-related reasons - e.g., cutting out meat helps lose or maintain weight," and "other (please specify)." Participants also indicated if they were currently vegetarian. In the interview portion of the study, individuals who had ever been vegetarian reported on what relationship, if any, vegetarianism had with their eating disorder by responding to the openended question: "In your opinion, what relationship did vegetarianism have to your eating disorder?" They also reported the age at which their eating disorder symptoms emerged to serve as a comparison to the age of onset of vegetarianism. This set of questions was developed for this study based in part on how questions about vegetarianism have been asked in the literature $(3,10,12)$.

Measures used to define eating disorder recovery status-The SCID (15) was used to determine lifetime and current eating disorders (AN, bulimia nervosa (BN), and Eating Disorder Not Otherwise Specified (EDNOS)). The Eating Disorder ExaminationQuestionnaire (EDE-Q; subscales: restraint, eating concern, weight concern, shape concern) (17) and portions of the Eating Disorders Longitudinal Interval Follow-up Evaluation (LIFE EAT II) (18) were used to collect data on the psychological and behavioral components of full recovery, respectively, and have psychometric support as valid measures $(17,18)$. Weight and height were measured (or self-reported for the minority who completed a phone interview) to determine BMI.

Demographic variables-Age, race/ethnicity, and parents' highest level of education in years were assessed via self-report. Parents' highest level of education attained was used as a proxy for socioeconomic status with, for example, 16 years reflecting four years of postsecondary schooling.

\section{Data Analysis}

For comparisons between those who had a history of an eating disorder $(n=93)$ and those who never had an eating disorder $(n=67)$, Chi square analyses were used for history of vegetarianism, current vegetarianism, and weight-related motivations as the primary motivation for vegetarianism. Analysis of covariance was used to compare age at becoming vegetarian since the eating disorder group was significantly older than the controls (see Table 1). Of note, the history of an eating disorder group was reduced from 96 to 93 because for three cases there were discrepancies in the questionnaire and interview data regarding vegetarianism (e.g., a participant endorsed having been vegetarian in the questionnaire, but not in the interview).

For comparisons among those at different stages of recovery from an eating disorder, groups were created using data from the SCID, the LIFE EAT II, the EDE-Q, and BMI following the guidelines proposed by Bardone-Cone and colleagues (13). There were 52 active eating disorder cases, 19 individuals meeting criteria for full recovery (i.e., no current eating disorder; minimum BMI of $18.5 \mathrm{~kg} / \mathrm{m}^{2}$; no binge eating, purging, or fasting in the prior three months; and within $1 S D$ of community norms on each of the EDE-Q subscales), and 15 meeting criteria for partial recovery, meaning all the criteria for full recovery except for psychological recovery (i.e., at least one EDE-Q subscale greater than $1 S D$ of age- matched norms). (For analyses involving stages of recovery, 86 of the 93 individuals contributed data by meeting criteria for one of the three stages of recovery). Of the 52 active cases, $17.3 \%$ ( $n$ $=9)$ currently met criteria for $\mathrm{AN}, 5.8 \%(n=3)$ for $\mathrm{BN}$, and 76.9\% $(n=40)$ for EDNOS, most with a bulimic-type presentation. The eating disorder recovery groups did not differ in their pattern of lifetime eating disorder diagnoses, the number of years since the emergence of the eating disorder symptoms, age or BMI at start of treatment, or current age (fully 
recovered: mean of $24.47 \pm 5.02$, partially recovered: mean of $23.53 \pm 5.80$, active eating disorder: mean of 23.20 \pm 4.43 ). These three groups were compared using Chi square analyses and analysis of variance. All statistical analyses were conducted using SPSS (version 19.0, 2010, IBM SPSS Inc., Armonk, NY).

\section{RESULTS}

A total of 160 women ranging in age from 16 to 40 years ( 93 with an eating disorder history, 67 without an eating disorder history) were included in the analyses comparing those with and without an eating disorder history. Data on the demographic variables for these samples are provided in Table 1.

Significantly more individuals with a history of an eating disorder reported ever having been a vegetarian, as well as currently being vegetarian, compared to individuals with no eating disorder history (see Table 2). For example, while about half of those with a history of an eating disorder reported having been vegetarian at some point, this was true for only about a tenth of the controls. While none of the controls who had ever been vegetarian reported becoming vegetarian primarily for weight-related reasons, almost half of those with an eating disorder history and some experience with vegetarianism reported being primarily motivated by wanting to lose or maintain weight (see Table 2). After controlling for current age, age at becoming vegetarian was not significantly different across groups, $F(1,53)=.88$, $p=.351$.

The women in the different stages of eating disorder recovery did not differ significantly in whether they had ever been vegetarian or in whether weight was the primary motivation for becoming vegetarian. However, they did differ in current vegetarianism status, with the active eating disorder group having the most current vegetarians (about one-third) and the fully recovered group having the fewest (about $5 \%$ ) (see Table 3). Age at becoming vegetarian was not significantly different across the recovery groups, $F(2,41)=2.38, p=$. 105.

Of individuals with an eating disorder history and experiences with vegetarianism, most $(68.1 \%)$ felt there was a relation between vegetarianism and their eating disorder. Those who reported there was a relation reported that being vegetarian helped them lose weight and maintain the eating disorder and provided another way to eliminate calories and feel in control. When comparing age at becoming vegetarian with age at the emergence of first eating disorder symptoms (both self-reports), most (59.6\%) reported that the starting age for vegetarianism came at least one year after the first eating disorder symptoms.

\section{DISCUSSION}

Women with a history of an eating disorder had significantly different vegetarianism-related experiences than women without an eating disorder history. Rates of ever having been vegetarian among those with an eating disorder history were similar to rates of vegetarianism in samples with AN $(7,8)$ and higher than rates among those without an eating disorder history. The finding regarding differences in motivations for becoming vegetarian is novel, never before having been examined in groups distinguished by a history of an eating disorder.

There were fewer differences related to vegetarianism when eating disorder recovery groups were examined. One exception was the higher rates of current vegetarianism in the active eating disorder group compared to the fully recovered group. This finding is in contrast to prior work that found vegetarianism still prominent among those recovered from $\mathrm{AN}(5,6)$. Differing definitions of recovery could explain this discrepancy. 
Most of the eating disorder sample with a history of vegetarianism reported that the eating disorder preceded the adoption of a vegetarian diet, as was found in some prior work (8). This finding is in line with research suggesting that vegetarian diets may be used to facilitate ongoing restriction rather than cause eating pathology $(3,19)$; however, prospective research is needed for etiological clarity.

In addition to longitudinal work, diagnostic differences (e.g., AN, BN) should also be considered in the future given the centrality of food restriction in AN and the finding that zinc deficiency resulting from vegetarianism among individuals with AN may influence eating disorder chronicity (20). Perhaps vegetarianism has a different meaning and is related differently to eating disorder tenacity among individuals with AN compared to those with BN.

The study findings have several practice-based implications. The finding that none of the controls who had ever been vegetarian were primarily motivated by weight-related reasons, in contrast to almost half of those with an eating disorder history, suggests the importance of attending to primary motives for becoming vegetarian. From an eating disorders perspective, individuals who are sincerely motivated to adopt vegetarianism for primarily non-weight reasons (e.g., ethics) may be less concerning than individuals with weight-based motives. Practitioners may also want to be mindful that vegetarianism is more likely adopted by individuals with preexisting disordered eating attitudes/behaviors, rather than being the cause of such pathology. Thus, when an adolescent begins a vegetarian diet or expresses interest in making this dietary choice, a close examination of her/his general eating attitudes/ behaviors may shed light on whether concern is warranted. Exploration of existing patterns of eating, attitudes about food, and the function vegetarianism may serve would shed light on the trajectory of the individual's relationship with food and whether vegetarianism may provide the individual with additional "good food"/"bad food" dichotomies that would simply her/his life. Collaboratively exploring whether a vegetarian lifestyle is an avoidance strategy for food may prompt a practitioner to challenge distorted thoughts surrounding "forbidden" foods. To the extent that becoming vegetarian signals more extreme weight control behavior $(9)$ and eating disorder chronicity $(8,20)$, practitioners would be welladvised to engage individuals in frank discussions regarding their eating habits. If an individual is firm in the decision to become vegetarian, she/he should be provided with psychoeducation and guidance in becoming a healthful vegetarian. Lastly, practitioners should take heed of the different rates of current vegetarianism between the fully recovered and active eating disorder groups. In cases when an individual working toward eating disorder recovery is still adhering to a vegetarian diet, practitioners may wish to engage her/ him in a discussion regarding how a restricted food repertoire, including vegetarianism, may affect the recovery process.

This exploratory study contributes to the very limited literature on vegetarianism and eating disorders and is novel in being the first to examine aspects of vegetarianism using a comprehensive definition of eating disorder recovery. The examination of multiple aspects of vegetarianism (i.e., history of, primary reason for, age at becoming vegetarian) is also a strength. Limitations include the cross-sectional design, the small sample size, the lack of differentiation of eating disorder diagnoses, the group differences in age and interview modality, the definition of vegetarianism, and the lack of a validated measure for assessing aspects of vegetarianism. Given the small number of vegetarians in the recovery groups, the findings for stages of recovery should be considered preliminary and need to be replicated in larger samples. Further, asking about vegetarianism using more precision (e.g., semivegetarian, vegan, etc.) would help identify if certain types of vegetarianism are more strongly connected to eating disorders or eating disorder recovery. 


\section{CONCLUSIONS}

Having a history of an eating disorder is associated with greater likelihood of having been (or currently being) vegetarian and having been primarily motivated by weight-related reasons. Results indicated that a history of vegetarianism is not associated with recovery status since percentiles having ever been vegetarian were similar across recovery groups; however, rates of current vegetarianism differed significantly by recovery status. Future research should examine vegetarianism and eating disorders/eating disorder recovery longitudinally in order to better understand how eating disorder symptoms and vegetarianism may propel each other over time and whether transitioning from a vegetarian diet may facilitate full recovery.

\section{References}

1. American Dietetic Association. Position of the American Dietetic Association: Vegetarian diets. J Am Diet Assoc. 2009; 109(7):1266-1282. [PubMed: 19562864]

2. Key TJ, Fraser GE, Thorogood M, et al. Mortality in vegetarians and nonvegetarians: Detailed findings from a collaborative analysis of 5 prospective studies. Am J Clin Nutr. 1999; 70(3):516S524S. [PubMed: 10479225]

3. Gilbody S, Kirk S, Hill A. Vegetarianism in young women: Another means of weight control? Int J Eat Disord. 1999; 26(1):87-90. [PubMed: 10349588]

4. Lindeman M, Stark K, Latvala K. Vegetarianism and eating-disordered thinking. Eat Disord: J Treat Prevent. 2000; 8(2):157-165.

5. Yackobovitch-Gavan M, Golan M, Valevski, et al. An integrative quantitative model of factors influencing the course of anorexia nervosa over time. Int J Eat Disord. 2009; 42(4):306-317. [PubMed: 19040269]

6. Hansson LM, Björck C, Birgegård A, Clinton D. How do eating disorder patients eat after treatment? Dietary habits and eating behaviour three years after entering treatment. Eat Weight Disord. 2011; 16(1):e1-8. [PubMed: 21727776]

7. Kadambari R, Gowers S, Crisp A. Some correlates of vegetarianism in anorexia nervosa. Int J Eat Disord. 1986; 5(3):539-544.

8. O'Connor MA, Touyz SW, Dunn SM, Beumont PJV. Vegetarianism in anorexia nervosa? A review of 116 consecutive cases. Med J Australia. 1987; 147(11-12):540-542. [PubMed: 3696039]

9. Perry C, McGuire M, Neumark-Sztainer D, Story M. Characteristics of vegetarian adolescents in a multiethnic urban population. J Adolescent Health. 2001; 29(6):406-416.

10. Trautmann J, Rau S, Wilson M, Walters C. Vegetarian students in their first year of college: Are they at risk for restrictive or disordered eating behaviors? Coll Student J. 2008; 42(2):340-347.

11. Tannhauser PP, Latzer Y, Rozen G, Tamir A, Naveh Y. Zinc status and meat avoidance in anorexia nervosa. Int J Adolescent Med Health. 2001; 13(4):317-326.

12. Klopp SA, Heiss CJ, Smith HS. Self-reported vegetarianism may be a marker for college women at risk for disordered eating. J Am Diet Assoc. 2003; 103(6):745-747. [PubMed: 12778048]

13. Bardone-Cone AM, Harney MB, Maldonado CR, et al. Defining recovery from an eating disorder: Conceptualization, validation, and examination of psychosocial functioning and psychiatric comorbidity. Behav Res Ther. 2010; 48(3):194-202. [PubMed: 19945094]

14. Reas DL, Williamson DA, Martin CK, Zucker NL. Duration of illness predicts outcome for bulimia nervosa: A long-term follow-up study. Int J Eat Disord. 2000; 27(4):428-434. [PubMed: 10744849]

15. First, MB.; Spitzer, RL.; Gibbon, M.; Williams, JB. Structured Clinical Interview for the DSM-IV Axis I disorders. Patient edition (SCID-I/P, version 2). New York State Psychiatric Institute, Biometrics Research Department; New York: 1995.

16. Curtis M, Comer L. Vegetarianism, dietary restraint and feminist identity. Eat Behav. 2006; 7(2): 91-104. [PubMed: 16600838]

17. Fairburn CG, Beglin SJ. Assessment of eating disorders: Interview or self-report questionnaire? Int J Eat Disord. 1994; 16(4):363-370. [PubMed: 7866415] 
18. Herzog DB, Sacks NR, Keller MB, Lavori PW, von Ranson KB, Gray HM. Patterns and predictors of recovery in anorexia nervosa and bulimia nervosa. J Am Acad Child Psy. 1993; 32(4):835-842.

19. Sullivan V, Damani S. Vegetarianism and eating disorders - Partners in crime? Eur Eat Disord Rev. 2000; 8(4):263-266.

20. Bakan R, Birmingham CL, Aeberhardt L, Goldner EM. Dietary zinc intake of vegetarian and nonvegetarian patients with anorexia nervosa. Int J Eat Disord. 1993; 13(2):229-233. [PubMed: 8477292] 


\section{Table 1}

Demographic characteristics of females with and without a history of an eating disorder

\begin{tabular}{lcccc}
\hline $\begin{array}{l}\text { Demographic } \\
\text { Variable }\end{array}$ & $\begin{array}{c}\text { No Eating } \\
\text { Disorder } \\
\text { History } \\
(\boldsymbol{n}=\mathbf{6 7})\end{array}$ & $\begin{array}{c}\text { Eating } \\
\text { Disorder } \\
\text { History } \\
(\boldsymbol{n}=\mathbf{9 3})\end{array}$ & Test Statistic & $p$-value \\
\hline $\begin{array}{l}\text { Age in years } \\
M \pm S D\end{array}$ & $19.46 \pm 1.88$ & $23.61 \pm 4.80$ & $t(158)=-6.71$ & $<0.001$ \\
$\begin{array}{l}\text { Ethnicity } \\
\text { Caucasian })\end{array}$ & $89.6 \%(n=60)$ & $92.5 \%(n=86)$ & $\mathrm{X}^{2}(1, N=160)=0.42$ & 0.519 \\
$\begin{array}{l}\text { (n) } \\
\text { Socioeconomic } \\
\text { status in years }\end{array}$ & $16.52 \pm 2.65$ & $16.70 \pm 2.84$ & $t(158)=-0.41$ & 0.682 \\
$M \pm S D$ & & & & \\
$\begin{array}{l}\text { Body Mass Index } \\
\text { in kg/m }\end{array}$ & $23.74 \pm 3.57$ & $21.93 \pm 3.84$ & $t(157)=3.02$ & 0.003 \\
$M \pm S D$ & & & & \\
\hline
\end{tabular}

${ }^{a} \mathrm{M}$ (means) and SD (standard deviations) are reported for age, socioeconomic status, and body mass index, which are continuous variables. Chi square statistics are reported for ethnicity with percentiles representing those identifying as Caucasian.

$b$ Defined by parents' highest level of education attained in years, which is used here as a proxy for socioeconomic status. 


\section{Table 2}

Proportions of females with and without a history of an eating disorder endorsing vegetarian experiences and weight-related motivations for vegetarianism

\begin{tabular}{lcccc}
\hline $\begin{array}{l}\text { Vegetarian } \\
\text { Variable }\end{array}$ & $\begin{array}{c}\text { No Eating } \\
\text { Disorder } \\
\text { History } \\
(\boldsymbol{n}=\mathbf{6 7})\end{array}$ & $\begin{array}{c}\text { Eating } \\
\text { Disorder } \\
\text { History } \\
(\boldsymbol{n}=\mathbf{9 3})\end{array}$ & $\mathrm{X}^{\mathbf{2}}$ & $p$-value \\
\hline Ever vegetarian & $11.9 \%^{a}(n=8)$ & $51.6 \%(n=48)$ & $\begin{array}{c}\mathrm{X}^{2}(1, N=160) \\
=26.94\end{array}$ & $<0.001$ \\
$\begin{array}{l}\text { Current } \\
\text { vegetarian }\end{array}$ & $6.0 \%(n=4)$ & $23.7 \%(n=22)$ & $\begin{array}{c}\mathrm{X}^{2}(1, N=160) \\
=8.95\end{array}$ & 0.003 \\
$\begin{array}{l}\text { Primary reason } \\
\text { for becoming } \\
\text { vegetarian was } \\
\text { weight-related }\end{array}$ & $0 \%(n=0)$ & $42.2 \%(n=19)$ & $\begin{array}{c}\mathrm{X}^{2}(1, N=53) \\
=5.27\end{array}$ & $\begin{array}{c}0.022 \\
(\text { Fisher's Exact } \\
\text { Test } p= \\
0.040)\end{array}$ \\
\hline
\end{tabular}

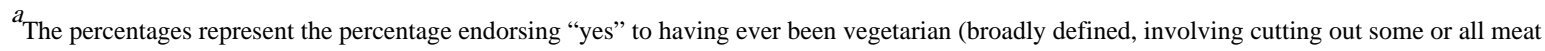
from one's diet) and "yes" to currently being vegetarian, as well as the percentages who reported that they became vegetarian primarily for weightrelated reasons.

${ }^{b}$ When one or more cells had an expected count less than 5, Fisher's Exact Test is reported in addition to Chi square statistics. 


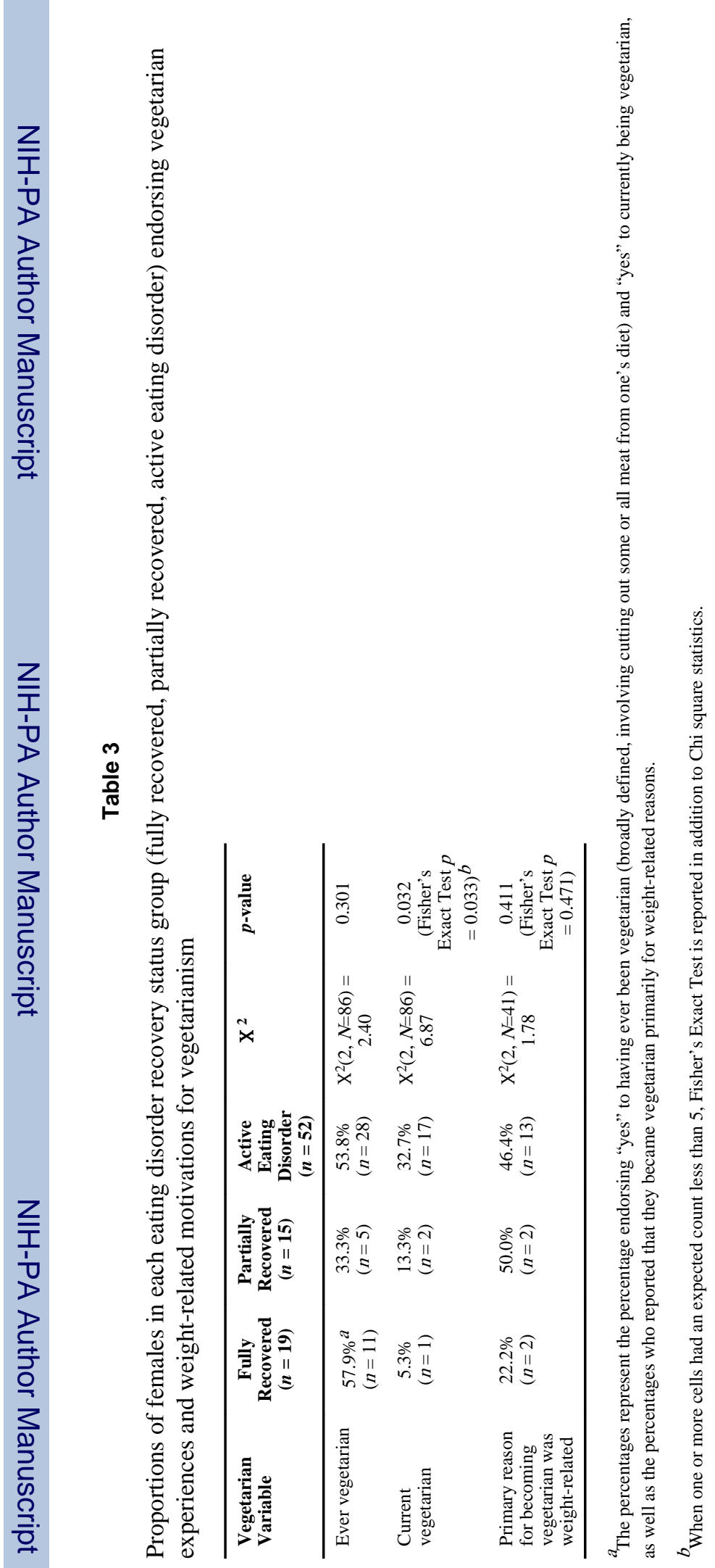

